Ulrike E. Nett, Markus Dresel

\title{
Selbstreguliertes Lernen in der Ganztagsschule
}

\section{Selbstreguliertes Lernen}

"Sollen Schüler ihre Kompetenzen allein erarbeiten?"In der Serie „Schulfrage" der ZEIT ONLINE vom Januar 2015 (Zeit Online, 2015) sieht sich ein Lehrer hin- und hergerissen zwischen den forderungen der Schulbehörde nach offenen Unterrichtskonzepten und seinen eigenen Erfahrungen, wonach direkte Unterrichtsmethoden deutlich effektiver sind. Diese Schulfrage und die damit verbundene mediale Diskussion verdeutlicht ein Dilemma, in welchem sich viele Lehrkräfte wiederfinden: Einerseits ist es erklärtes Ziel, unsere Schülerinnen und Schüler zu selbstständigem und lebenslangem Lernen zu befähigen (2. B. Perels 2011, 5. 3-4), besonders in einer Zeit, in der sich die Anforderungen und Herausforderungen des Berufslebens ständig verändern. Somit ist die fähigkeit zum selbstregulierten Lernen selbst zum Bildungsziel geworden. Um dies zu erreichen, solite den Schülerinnen und Schülern bereits in der Schule ausreichend Freiraum zum selbstregulierten Lernen gegeben werden. Andererseits tragen Lehrerinnen und Lehrer jedoch auch Verantwortung dafür, dass ihre Schülerinnen und Schüler Fachwissen und Fachkompetenzen erwerben. Dies geschieht oft effektiver und kontrollierter in Lernumgebungen, die stark durch die Lehrkraft gesteuert werden (vgl. z. B. Kirschner, et. al. 2006).

Im vorliegenden Kapitel wird zunächst der Begriff des selbstregulierten Lernens geklärt und umrissen, welche Komponenten selbstreguliertes Lernen umfasst. Im Anschluss daran werden Ergebnisse der empirischen forschung vorgestellt, welche einerseits die Chancen selbstregulierten Lernens betonen und andererseits die Herausforderungen hervorheben, die damit in der Schule einhergehen. Abschlie- 
Bend wird vorgestellt, wie selbstreguliertes Lernen an der Schule gefördert werden kann und welche besonderen Chancen hierfür an Ganztagsschulen bestehen.

\section{Definition selbstregulierten Lernens}

Der Begriff des selbstregulierten Lernens wurde bereits auf unterschiedliche Art und Weise definiert (vgl. z. B. Zimmerman 2001; Winne/Hadwin 1998; Pintrich 2005). Götz und Nett orientieren sich an der Begrifflichkeit selbst und bemühen sich so, den gemeinsamen Kern der meisten Definitionen zu vereinen. Sie beschreiben selbstreguliertes Lernen als „[...] eine Form des Erwerbs von Wissen und Kompetenzen, bei der Lerner sich selbständig und eigenmotiviert Ziele setzen sowie eigenständig Strategien auswählen, die zur Erreichung dieser Ziele führen und durch Bewertung von Erfolgen bezüglich der Reduzierung der Ist-Soll-Differenz Ziele und Aktivitäten im Hinblick auf eine Erreichung des Soll-Zustands prozessbegleitend modifizieren und optimieren". (Götz und Nett 2017, S. 146)

Diese recht technische Definition umfasst den vollständigen Lernprozess. Es wird detailliert aufgelistet, in welchen Einzelschritten des Lernprozesses Selbstregulation stattfinden kann, nämlich in der Definition der (Lern-)Ziele, der Wahl der (Lern-) Strategien zur Zielerreichung, der Bewertung des Lernprozesses und gegebenenfalls in der Anpassung des Lernprozesses.

Lehrerinnen und Lehrer übernehmen Verantwortung dafür, ihre Schülerinnen und Schüler zu eigenständigen und motivierten Lernenden auszubilden sowie ihnen fachliche Kompetenzen und fachliches Wissen zu vermitteln. Mit der obigen Definition stellt sich nun eine Reihe von Fragen: Inwiefern sind Lehrerinnen und Lehrer verantwortlich für die (Lern-)Ziele? In welchem Maße müssen sie diese vorgeben? Welche Freiräume können sie ihren Schülerinnen und Schülern in der Zielsetzung geben? Entsprechende Fragen stellen sich für die Wahl der (Lern-)Strategien, die Bewertung und die eventuelle Optimierung des Lernprozesses. Bereits Schiefele und Pekrun (1996) betonen, dass ein Lemprozess stets teils selbst- und teils fremdreguliert ist. Wirkliches Lernen kann niemals ohne ein gewisses Maß an Eigenständigkeit stattfinden, aber in fast allen Situationen findet auch Fremdsteuerung statt. Lehrerinnen und Lehrern obliegt nun die Aufgabe, hier eine ausgewogene Balance zu finden, in der sie den Schülerinnen und Schülern einerseits möglichst große Freiräume zugestehen, um eigene Kompetenzen im selbstregulierten 
Lernen zu erwerben und auszutesten und andererseits ausreichend Hilfestellung und Unterstützung bieten, um die Kompetenzen zum selbstregulierten Lernen zu erweitern und den Lernerfolg zu sichern.

\section{Komponenten selbstregulierten Lernens}

Boekaerts (1999) schlägt ein Drei-Schichtenmodell selbstregulierten Lernens vor, in welchem sie drei wesentliche Komponenten unterscheidet. Diese drei Komponenten sind hierarchisch geordnet und essentiell für erfolgreiches selbstreguliertes Lernen. Sie unterscheidet (1) die Regulation des Verarbeitungsmodus, (2) die Regulation des Lernprozesses und (3) die Regulation des Selbst (vgl. Abbildung 1).

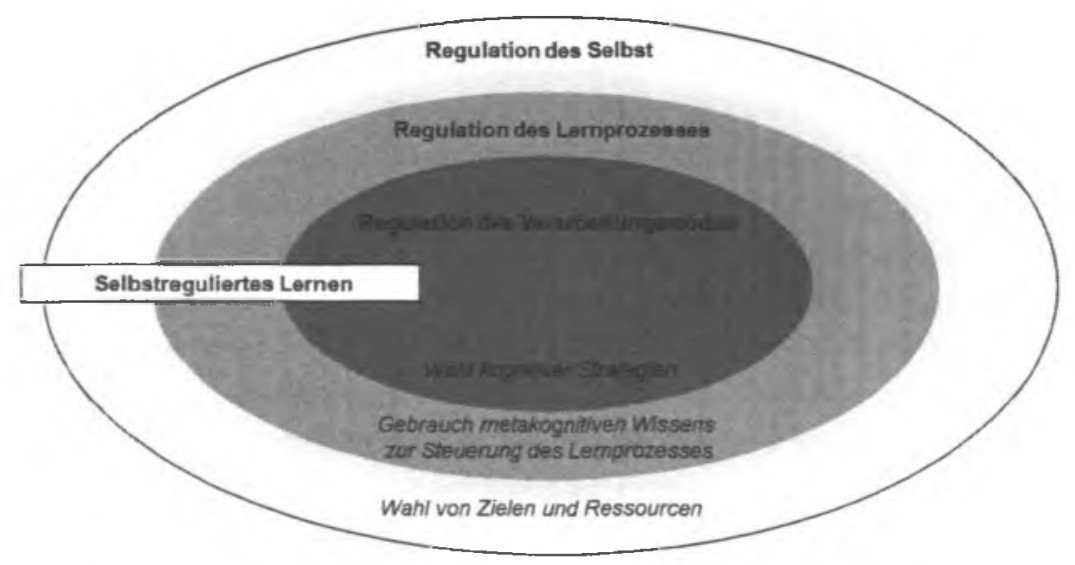

Abb. 1. Drei-Schichtenmodell des selbstregulierten Lernens nach Monique Boekaerts (1999, S. 449)

In den Mittelpunkt stellt Boekaearts (1999) die Regulation des Verarbeitungsmodus. Dies beinhaltet insbesondere die Wahl kognitiver Strategien (vgl. Dunlosky et. al. 2013), Lernstrategien wie z. B. Mnemotechniken oder Highlighting, das Erstellen von Mindmaps oder das Formulieren und Lösen eigener Übungsaufgaben. Um eine sinnvolle Auswahl treffen zu können, müssen Schülerinnen und Schüler über ein eigenes Repertoire an Lernstrategien verfügen. Darüber hinaus sollten sie 
in der Lage sein zu beurteilen, in welcher Lernsituation (abhängig z. B. vom Lerninhalt, der Aufgabenstellung und den Zielen) welche Lernstrategie angemessen ist. Lehrerinnen und Lehrer sind hier in der Verantwortung, ihren Schülerinnen und Schülern einerseits ein reichhaltiges Repertoire an Lernstrategien zu vermitteln, sie darüber hinaus aber auch darin zu unterstützen, die jeweils passenden Lernstrategien auszuwählen.

Insgesamt kann der Lernprozess nur gelingen, wenn der Einsatz der einzelnen kognitiven Strategien sinnvoll organisiert wird. Die Regulation des Lernprozesses beinhaltet den Einsatz metakognitiver Strategien, insbesondere die Planung, das Monitoring und die Evaluation des Lernprozesses. Während Schülerinnen und Schüler noch relativ häufig zum Einsatz spezifischer kognitiver Strategien angeregt werden, wird ihnen die Regulation des Lernprozesses durch metakognitive Strategien oft durch die Lehrkraft abgenommen. Beispielsweise unterstützen Lehrkräfte bei der Vorbereitung auf eine Klassenarbeit in der Planung, aber auch im Monitoring, indem sie klare Lernziele setzen und Übungsklassenarbeiten erstellen und korrigieren. Dabei die für jede Schülerin und jeden Schüler richtige Passung zwischen Fremdregulation und Freiheit zur Selbstregulation zu finden, erweist sich oft als große Herausforderung.

Die Regulation des Lemprozesses wiederum setzt eine Regulation des Selbst voraus. Ein Lernprozess kann nur effektiv geplant, überwacht und gegebenenfalls optimiert werden, wenn die Ziele den Fähigkeiten und Ressourcen des Lernenden entsprechend festgesetzt werden und auch übergeordnete individuelle Bedingungen wie z.B. die Motivation des Lernenden oder die Emotionen reguliert werden. Die Fähigkeit, nicht nur den Lernprozess selbst, sondern auch die eigene Motivation und Emotionen zu regulieren, ist dabei essentiell. Erst Lernende, die trotz motivationaler und emotionaler Konflikte einen Lemprozess initiieren, aufrechterhalten und erfolgreich abschließen können, regulieren ihr Lernen im umfassenden Sinne selbst.

Bedeutsam ist in diesem Modell des selbstregulierten Lernens (Boekaerts 1999), dass die unterschiedlichen Bereiche nicht für sich stehen, sondern sich gegenseitig bedingen. Eine effektive und zielgerichtete Auswahl von Lernstrategien kann nur gelingen, wenn sowohl die Anforderungen des Lernprozesses als auch die individuelien Bedingungen und persönlichen Ziele des Lernenden berücksichtigt wer- 
den (vgl. Dunlosky et al. 2013). Andererseits kann der Lernprozess nur erfolgreich sein, wenn diejenigen kognitiven und metakognitiven Strategien bekannt sind und angewendet werden können, die den Zielen und der Motivation des Lernenden entsprechen (vgl. Boekaerts 1999).

\section{Empirische Befunde zu selbstreguliertem Lernen}

Aus dem Modell von Boekaerts (1999) kann geschlossen werden, dass selbstreguliertes Lernen nicht nur selbst Bildungsziel ist, sondern auch einen positiven Einfluss auf den Lernerfolg haben kann. Im Folgenden werden empirische Befunde vorgestellt, die einen Einblick in das aktuelle Lernverhalten und die damit verbundene Selbstregulation von Schülerinnen und Schülern geben sollen.

\section{Selbstreguliertes Lernen im schulischen Alltag}

Nett, Goetz, Hall und Frenzel (2012) begleiteten in einer Studie 70 Schülerinnen und Schüler der 11. Jahrgangsstufe über die Dauer von zwei Wochen direkt vor einer Klassenarbeit in Mathematik. Über kurze digitale Fragebögen wurden die Schülerinnen und Schüler sechsmal am Tag dazu befragt, ob sie sich aktuell mit der Vorbereitung auf die Klassenarbeit befassen. Darüber hinaus wurde die Nutzung der metakognitiven Strategien Planung, Monitoring und Evaluation erfasst. Es zeigte sich. dass Schülerinnen und Schüler sich durchaus bewusst und zu sinnvollen Zeiten mit der Klassenarbeit beschäftigen. Insgesamt steigt die (meta-)kognitive Beschäftigung mit der Klassenarbeit an, je näher die Klassenarbeit zeitlich rückt. Sowohl die Intensität der Beschäftigung mit der Klassenarbeit als auch der Anstieg in der Beschaftigungsintensität hin zur Klassenarbeit hängen positiv mit der Leistung in der Klassenarbeit zusammen. Differenziert man die Beschäftigung mit der Klassenarbeit stärker und fokussiert auf die Nutzung der einzelnen metakognitiven Strategien Planung. Monitoring und Evaluation, so zeigt sich ebenfalls für jede der einzelnen Strategien ein Anstieg hin zur Klassenarbeit, die Note wird jedoch nur (und schwach) durch Monitoring vorhergesagt. Diese Studie gibt somit Hinweise darauf, dass Schülerinnen und Schüler zwar Strategien des selbstregulierten Lernens durchaus anwenden, die Nutzung dieser Strategien im Schulalitag jedoch nur wenig zum Schulerfolg beiträgt. Ein Grund hierfür könnte sein, dass der Schut- 
alltag und auch die Vorbereitung auf die Klassenarbeit stark external durch die Lehrkraft reguliert werden, eine eigenständige Regulation des Lernprozesses somit kaum, und dies auch nur zu geringem Maße bei Monitoring, mit Leistung assoziiert werden kann (Nett et al. 2012, S. 12). Die Ergebnisse dieser Einzelstudie werden von Metaanalysen von Dent und Koenka (2016) untermauert. Die Autoren fassen die Ergebnisse von 61 Studien zusammen und zeigen, dass der Zusammenhang zwischen kognitiven und metakognitiven Strategien des selbstregulierten Lernens und der Lernleistung meist eher schwach ausgeprägt ist. Allerdings hängt die Stärke des Zusammenhangs unter anderem stark von der untersuchten Strategie ab, beispielsweise scheint die Nutzung metakognitiver Strategien als Strategiebündel einen stärkeren Einfluss auf die Leistung zu haben als die Nutzung von Einzelstrategien.

Zusammenfassend kann aus den Studien geschlossen werden, dass Schülerinnen und Schüler über Fähigkeiten zum selbstregulierten Lernen verfügen und diese auch einen Einfluss auf die Lernleistung haben. Jedoch sind sowohl die Anwendung von Selbstregulationsstrategien als auch die Wirkung der Strategien im Schulalltag vermutlich eher schwach ausgeprägt und werden von weiteren Faktoren beeinflusst. Aus theoretischer Perspektive ist anzunehmen, dass die Gestaltung der Lernumgebung und die Freiheitsgrade, welche durch die Lehrkraft gegeben werden, hierfür von Bedeutung sind.

\section{Effekte von Förderprogrammen zu selbstreguliertem Lernen}

Die bisher vorgestellte Studie von Nett und Kollegen (2012) sowie die Studien, die in der Metaanalyse von Dent und Koenka (2016) zusammengefasst wurden, beschäftigen sich mit der Frage, wie Schülerinnen und Schüler ihr Lernen im Schulalltag selbst regulieren. Ejne weitere Perspektive auf die Effektivität selbstregulierten Lernens bieten Interventionsstudien, in welchen untersucht wird, auf welche Art und Weise selbstreguliertes Lernen bei Schülerinnen und Schülern unterstüzt werden kann. Dresel und Haugwitz (2008) beispielsweise führten mit 151 Schülerinnen und Schülern der 6 . Jahrgangsstufe ein computerbasiertes Mathematiktraining während des Regelunterrichts durch. In einem quasiexperimentellen Design unterschieden sie drei Gruppen. Während die Kontrollgruppe nur das Mathematiktraining erhielt, erhielt eine erste Experimentalgruppe attributionsorientierte Rück- 
meldung zur Förderung der Motivation und eine zweite Experimentalgruppe zusätzlich zu dieser Rückmeldung ein Training zur Förderung der Anwendung metakognitiver Strategien (Planung, Zielsetzung, Monitoring, Selbstevaluation). Es zeigten sich Trainingseffekte in Bezug auf die Motivation in beiden Experimentalgruppen, in der Experimentalgruppe, die zusätzlich noch ein Selbstregulationstraining erhielt, zeigte sich auch eine verbesserte Nutzung von metakognitiven Strategien. Besonders hervorzuheben ist darüber hinaus, dass beide Experimentalgruppen einen stärkeren Wissenszuwachs aufwiesen als die Kontrollgruppe, wobei der Wissenszuwachs in der Selbstregulationsgruppe am größten war.

Inzwischen beschäftigen sich zahlreiche Studien mit der Frage, auf welche Weise selbstreguliertes Lernen in der Schule effektiv gefördert werden kann und ob durch dessen Förderung ein positiver Einfluss auf das Lernverhalten, die Motivation, die lernbezogenen Emotionen und insbesondere auf den Lernerfolg der Schülerinnen und Schüler erzielt werden kann. Dabei werden sehr unterschiedliche interventionsdesigns genutzt. Dennoch zeigt eine Reihe von Metaanalysen (vgl. Hattie et. al. 1996; Dignath et. al. 2008; Dignath/Büttner 2008; Donker-Bergstra et. al. 2014), unter welchen Voraussetzungen Interventionsprogramme zur Förderung selbstregulierten Lernens besonders effektiv sind.

Bereits die Metaanalyse von Hattie et al. (1996) belegt, dass Interventionsprogramme zur Förderung selbstregulierten Lernens einen positiven Einfluss auf Leistung, Lernverhalten und Affekt haben. Neuere Metaanalysen bestätigen dieses $\mathrm{Er}$ gebnis und belegen sogar stärkere Effekte (vgl. Dignath/Büttner 2008, DonkerBergstra et al. 2014). Dies gilt sowohl für Interventionen im Primar- als auch im Sekundarschulbereich (vgl. Dignath \& Büttner 2008; Donker-Bergstra et al. 2014). Insgesamt gibt es Hinweise, dass Förderprogramme, in denen vorwiegend metakognitive Strategien (z. B. Hattie et al. 1996; Dignath/Büttner 2008; Donker-Bergstra 2014) und motivationale Strategien (z. B. Dignath/Büttner 2008) gefördert werden, wirkungsvoller sind, insbesondere dann, wenn sie in einen konkreten Lernkontext eingebettet werden (z. B. Hattie et al. 1996). Eine Studie mit jüngeren Schülerinnen und Schülern indiziert zudem, dass Förderprogramme nochmals optimiert werden können, wenn bereits in der Grundschule die Mechanismen selbstregulierten Lernens modelliert werden ( $z$. B. indem ein altersgerecht aufbereitetes Modell selbstreguliertes Lernen im Fachunterricht thematisiert und das entsprechende Lernver- 
halten erläutert wird) und auf diese Weise Metawissen über selbstreguliertes Lernen entwickelt wird (vgl. Stöger/Sonntag/Ziegler 2014).

\section{Förderung selbstregulierten Lernens in der Schule}

Aus den bisherigen Überlegungen und empirischen Befunden lässt sich ableiten, dass selbstreguliertes Lernen einerseits Bildungsziel in Vorbereitung auf ein eigenständiges und mündiges Leben ist, andererseits aber auch das Lernen von Fachinhalten an der Schule selbst erleichtern kann. Zahlreiche empirische Studien belegen, dass Programme zur Förderung selbstregulierten Lernens an der Schule erfolgreich sein können und einen positiven Einfluss auf das Lernverhalten, die Motivation, die lernbezogenen Emotionen und insbesondere den Lernerfolg haben können. Auch Lehrkräfte legen Wert auf die Förderung selbstregulierten Lernens und die Gestaltung von offenen Lernumgebungen. Dies stellten Dignath-van Ewijk und van der Werf (2012) in einer Befragung von Grundschullehrerinnen und Grundschullehrern fest. Während jedoch die Mehrheit der Lehrkräfte, die das selbstregulierte Lernen der Schülerinnen und Schüler fördern möchten, angeben, offene und Autonomie gewährende Lernumgebungen zu gestalten, schulen nur wenige dieser Lehrerinnen und Lehrer die Schülerinnen und Schüler explizit in der Nutzung von Strategien des selbstregulierten Lernens. Bereits die Eingangsfrage legt jedoch nahe, dass es gerade hier auf die passende Balance ankommt: Zum einen sollten die Freiheitsgrade der Lernumgebung an die Fähigkeiten der Schülerinnen und Schüler zum selbstregulierten Lernen sowie an ihre spezifischen Voraussetzungen angepasst sein ( $\mathrm{vgl}$. Sierens et al. 2009). Zum anderen sollten die Fähigkeiten der Schülerinnen und Schüler zur Selbstregulation gemäß den Anforderungen der Lernumgebung geschult werden.

\section{Herausforderungen bei der Förderung selbstregulierten Lernens}

Die oben beschriebene Balance ist zentral für das fördermodell selbstregulierten Lernens von Götz und Nett (2017, S. 172) bzw. für die leicht adaptierte Version von Nett und Götz (in press, vgl. Abbildung 2). Entsprechend dieses Modells ist es wichtig, das Wissen und die Kompetenzen zu Aspekten selbstregulierten Lernens und die Freiheit dazu in der Gestaltung von Lernumgebungen aufeinander abzustim- 


\section{Modell zur Förderung selbstregulierten Lernens}

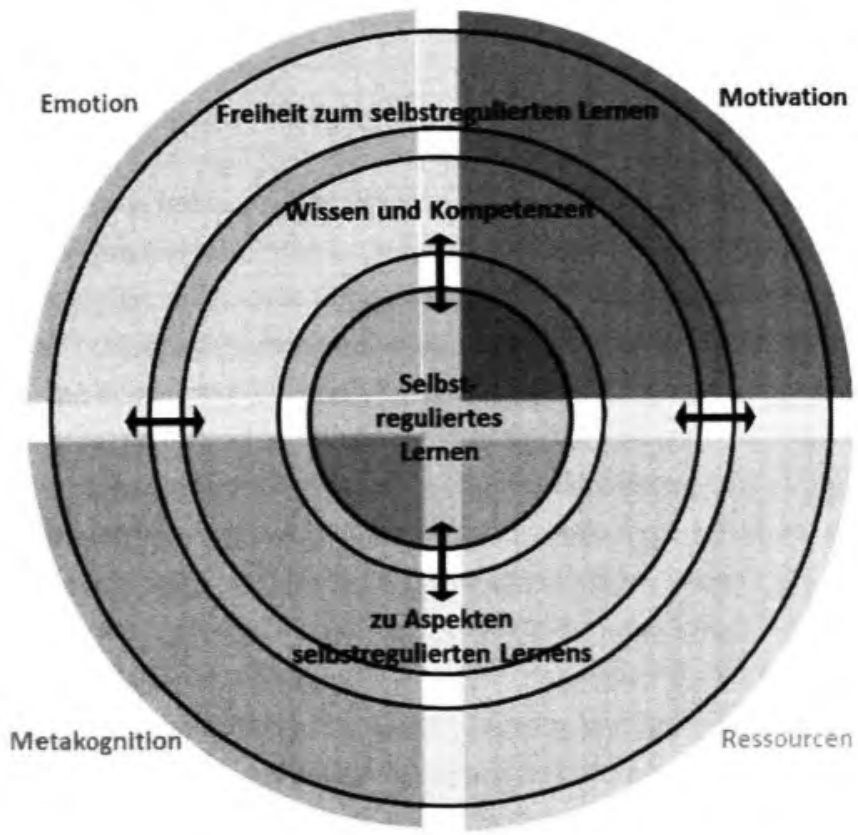

Abb. 2: Fördermodell selbstregulierten Lernens. Nett, U.E./Götz, T.: Selbstreguliertes Lernen. In: Detlef Urhahne/Markus Dresel/Frank Fischer (Hrsg.) (2019): Psychologie für den Lehrberut. Springer-Verlag Berlin Heidelberg.

men. Darüber hinaus sollten Lehrkräfte individuelle Voraussetzungen der Lernenden berücksichtigen. Hier betonen Götz und Nett die Bedeutung der Motivation, der damit verbundenen Emotionen sowie die Metakognition über selbstregulierende Prozesse und entsprechende Ressourcen. Sowohl Motivation als auch Emotionen können von Lehrerinnen und Lehrern gelenkt werden, indem sie explizit die Bedeutung eigenständigen Lernens vermitteln und Gelegenheiten schaffen, bei denen sich ihre Schülerinnen und Schüler als selbstwirksam erleben (vgl. hierzu auch Götz und Nett 2017, S. 172; sowie Nett und Götz, in press). Dass Förderprogramme zum selbstregulierten Lernen besonders dann wirksam sind, wenn auch metakognitives Wissen darüber vermittelt wird, wurde bereits im vorangehenden 
Abschnitt thematisiert. Eng mit den drei bereits genannten Bereichen verwoben ist die Berücksichtigung individueller Ressourcen. Selbstreguliertes Lernen kann, insbesondere bei unerfahrenen Lernenden, im Vergleich zu stark external regulierten Lernprozessen sogar verstärkt besondere Ressourcen beanspruchen (Nett und Götz, in press). So entsteht das eingangs beschriebene Dilemma vieler Lehrkräfte. Ihnen fehlen oft wichtige Ressourcen wie (flexible) Lern- und Übungszeiten, günstige Strukturen während der Hausaufgaben, fächerübergreifende Kooperationsmöglichkeiten und Strukturen, u.v.m. Gerade hier bietet die Ganztagsschule besondere Chancen, die im Folgenden skizziert werden. Dabei werden nur besonders offensichtliche Chancen skizziert, je nach Konzept der Ganztagsschule bieten sich gewiss noch deutlich mehr Möglichkeiten.

\section{Chancen für mehr selbstreguliertes Lernen an der Ganztagsschule}

Ganztagsschulen sind nach sehr individuellen, unterschiedlichen Konzepten aufgebaut (vgl. hierzu z. B. Appel/Rutz 2009), von daher ist es kaum möglich, von „der Ganztagsschule“ zu sprechen. Eine besonders wichtige Ressource bieten jedoch alle Ganztagsschulen - Zeit. Schülerinnen und Schüler verbringen über die in den Bildungs- und Unterrichtsplänen meist relativ klar festgelegte Unterrichtszeit hinaus auch einen großen Teil des Nachmittags in der Ganztagsschule. Diese Zeit ist nicht nur schulischem Lernen vorbehalten - die Schülerinnen und Schüler sollen sich auch erholen, ihren Interessen nachgehen, spielen, Sport machen, musizieren und vieles mehr - sie kann und soll jedoch auch in Teilen den Hausaufgaben und dem schulischen Üben zugutekommen. In einigen Ganztagsschulen (vgl. z. B. Fontein 2018, S. 168, für das Beispiel des Elsa-Brändström-Gymnasiums, Oberhausen) sind Hausaufgaben durch strukturierte übungszeiten ersetzt worden. Diese Zeiten müssen, je nach Konzept, nicht notwendigerweise ausschließlich nachmittags stattfinden. So können über diese zusätzliche Zeitressource in einem gut abgestimmten Ganztagskonzept neue Chancen zur Förderung selbstregulierten Lernens entstehen.

- Das eingangs beschriebene Dilemma von Lehrkräften zwischen stark geführten und offenen Unterrichtskonzepten kann auf diese Weise aufgelöst werden. Lehrkräfte können bestimmte Inhalte in geführten Lehrformaten anbieten und zu anderen Zeiten offene Lernumgebungen gestalten. Insbesondere an Ganztages- 
schulen können sie Übergänge schaffen, in welchen sie ihre Schülerinnen und Schüler schrittweise an das eigenständige Lernen in offenen Lernumgebungen heranführen. Wichtig ist, dass sie dabei nicht die Kontrolle über das tatsächliche Lernen ihrer Schülerinnen und Schüler verlieren bzw. der Verantwortung für deren Lernerfolg nicht mehr gerecht werden (vgl. Fontein 2018).

- Wichtiger Bestandteil selbstregulierten Lernens ist die eigenständige Setzung der Lernziele - doch im Unterrichtsalltag an Halbtagsschulen sind diese meist durch den Lehrplan definiert. An Ganztagsschulen kann den Schülerinnen und Schülern außerhalb der Unterrichtszeit beispielsweise im Rahmen von Projektarbeit deutlich einfacher die Möglichkeit gegeben werden, sich eigene Lernziele und Lernaufgaben zu setzen, die möglicherweise auch wenig oder nichts mit den Unterrichtszielen zu tun haben. Insbesondere in solchen Lernumgebungen können Schülerinnen und Schüler ganz neue Lernformen entdecken.

- Lehrkräfte können in diesen freien Bereichen bzw. offenen Lernumgebungen unterschiedliche Bewertungs- und Rückmeldemöglichkeiten nutzen und so die Schülerinnen und Schüler ganz individuell in ihrem Lernprozess unterstützen (vgl. z. B. Winter 2015).

- Im Rahmen dieser zusätzlich gewonnenen Zeiten wird auch eine echte Möglichkeit für fachübergreifende Lernumgebungen ermöglicht, da dies nicht auf Kosten eines bestimmten Faches bzw. der fachspezifischen Ziele gehen muss. Bei einer geschickten Einteilung der betreuenden Lehrkräfte, Pädagoginnen und Pädagogen im Ganztag können ganz neue Kooperationen entstehen.

- Viele Ganztagsschulen haben zumindest in Teilen auch jahrgangsübergreifende Strukturen wie z. B. jahrgangsübergreifende Betreuung am Nachmittag, teilweise auch Betreuung durch ältere Schülerinnen und Schüler. Diese Strukturen verhelfen zu vielfältigen Möglichkeiten zur Vertiefung der Kompetenzen im selbstregulierten Lernen, sei es durch das Lernen am Modell, durch das eigene Unterstützen anderer Schülerinnen und Schüler oder durch das Lernen in Gruppen. Auch so werden Lernstrategien trainiert.

Nur wenige Konzepte von Ganztagsschulen sehen vor, dass die Schülerinnen und Schüler zu jeder Zeit von ihren Lehrkräften betreut werden. Meist sind ganz unterschiedliche Personen - Lehrkräfte, Pädagoginnen und Pädagogen, Erzieherinnen und Erzieher, Studierende, manchmal auch Schülerinnen und Schüler einer höhe- 
ren Jahrgangsstufe, Eltern und viele mehr - in die Betreuung der Schülerinnen und Schüler eingebunden. Dies birgt die Gefahr, dass sich jede einzelne Person nur für ihren Bereich zuständig fühlt und insbesondere die Fachlehrkräfte nur wenig mit der Betreuung am Nachmittag in Berührung kommen. Es bietet jedoch auch die Chance, dass alle Beteiligten ihre unterschiedlichen Perspektiven auf das Lernen einbringen können und die Schülerinnen und Schüler davon profitieren. Hierfür sind eine gute Koordination und effektive Absprachen notwendig. Wichtig ist bei allem, dass die theoretischen Überlegungen und empirischen Ergebnisse berücksichtigt werden. Die Fähigkeit zum eigenständigen Lernen entsteht nicht notwendigerweise von selbst, sondern solite auch trainiert werden. Ganztagsschulen können in besonderem Maße dafür Lerngelegenheiten bieten.

\section{Literatur}

Appel., S./Rutz, G. (2009): Handbuch Ganztagsschule. Praxis, Konzepte, Handreichungen. Schwalbach/Ts.

Boekaerts, M. (1999): Self-regulated learning: Where we are today. International Journal of Educational Research, 31(6), 5. 445-457.

Dent, A. L./Koenka, A. C. (2016): The relation between self-regulated learning and academic achievement across childhood and adolescence: a meta-analysis. Educational Psychology Review, 28(3), S. 425-474.

Dignath C./Büttner, G. (2008): Components of fostering self-regulated learning among students. A meta-analysis on intervention studies at primary and secondary school level. Metacognition and Learning, 3(3), 5. 231-264.

Dignath C./Büttner, G./Langfeldt, H. P. (2008): How can primary school students learn self-regulated learning strategies most effectively? A meta-analysis on self-regulation training programmes. Educational Research Review, 3, S. 101-129.

Dignath-van Ewijk, C./van der Werf, G. (2012): What teachers think about self-regulated learning: Investigating teacher beliefs and teacher behavior of enhancing students' self-regulation. Educational Research International, doi: 10.1155/2012/741713.

Donker-Bergstra, A./De Boer, H., Kostos/D., Dignath-van Ewijk, C./van der Werf, C. (2014): Effectiveness of learning strategy instruction on academic performance: a meta-analysis. Educational Research Review, 11, S. 1-26.

Dresel, M./Haugwitz, M. (2008): A computer-based approach to fostering motivation and self-regulated learning. The Journal of Experimental Education, 77(1), 5. 3-18. 
Dunlosky, J./Rawson, K. A./Marsh, E. J./Nathan, M. J./Willingham, D. T. (2013): Improving students' learning with effective learning techniques promising directions from cognitive and educational psychology. Psychological Science in the Public interest, 14(1), 5. 4-58.

Ertl, H. (2006): Educational standards and the changing discourse on education: The reception and consequences of the PISA study in Germany. Oxford Review of Education, 32, S. 619-634.

Fontein, B. (2018): Auf dem Weg zu einer neuen Lernkultur. In: S. Maschke/G. SchulzGade/L. Stecher (Hg.): Jahrbuch Ganztagsschule, (S. 165-170). Frankfurt/M.

Götz, T./Nett, U. E. (2017): Selbstreguliertes Lernen. In: T. Götz (Hg.): Emotion, Motivation und selbstreguliertes Lernen (S. 143-183). Paderborn.

Hattie, J. A./Biggs, J./Purdie, N. (1996): Effects of learning skills interventions on student learning: A meta-analysis. Review of Educational Research, 66(2), S. 99-136.

Kirschner, P. A./Sweller, 1./Clark, R. E. (2006): Why minimal guidance during instruction does not work: An analysis of the failure of constructivist, discovery, problem-based, experiential, and inquiry-based teaching. Educational psychologist, 41(2), S. 75-86.

Nett, U. E./Goetz, T. (in press): Selbstreguliertes Lernen. In: D. Urhahne/M. Dresel/F. Fischer ( $\mathrm{Hg}$.): Psychologie für den Lehrerberuf. Heidelberg.

Nett, U. E./Götz, T./Hall, N. C./Frenzel, A. C. (2012): Metacognitive strategies and test performance: An experience sampling analysis of students' learning behavior. Education Research International. DOI.10.1155/2012/958319.

Perels, F. (2011): Selbstregulierts Lernen. Wiesbaden: $1 \mathrm{Q}$ kompakt. Verfügbar unter https:// kultusministerium.hessen.de/sites/default/files/media/selbstreguliertes_lernen. pdfhttps://kultusministerium.hessen.de/sites/default/files/media/selbstreguliertes_ lernen.pdf 